\title{
Research on Knowledge Resources Investment Decisions in Cooperated New Product Development
}

\author{
Jiangbo Zheng, Yanan Wang \\ School of Management, Jinan University, Guangzhou, China \\ Email:wangyanan94@126.com
}

How to cite this paper: Zheng, J.B. and Wang, Y.N. (2018) Research on Knowledge Resources Investment Decisions in Cooperated New Product Development. Journal of Service Science and Management, 11, 101-115.

https://doi.org/10.4236/jssm.2018.111010

Received: January 10, 2018

Accepted: February 24, 2018

Published: February 27, 2018

Copyright $\odot 2018$ by authors and Scientific Research Publishing Inc. This work is licensed under the Creative Commons Attribution International License (CC BY 4.0).

http://creativecommons.org/licenses/by/4.0/

\begin{abstract}
Existed researches on resources investment decisions in cooperated new product development always omit the enterprise's knowledge level, often ignore the impact of knowledge resources on the value of new products and cooperation. Based on the existing research on transactional behavior among supply chain enterprises, most of them consider the transaction of physical substances and less consider knowledge resources, this paper takes the cooperated $\mathrm{R} \& \mathrm{D}$ among enterprises as an example to explore the optimal resource investment decisions of cooperative participants under the condition that both parties invest knowledge resources. By constructing the Stackelberg game model, we discussed the optimal cooperative decisions of manufacturers and suppliers, and the influences of different factors on cooperative decision-making.
\end{abstract}

\section{Keywords}

Supply Chain, Cooperated New Product Development, Knowledge Resources, Knowledge Transaction

\section{Introduction}

The new product development (hereinafter referred to as "cooperated development") has become an effective means to highlight the core competitiveness of enterprises and enhance their competitive power [1]. The process of resources investment in cooperated development not only occupies most part cost, and directly affect the success or failure [2], so the resources optimization problem has attracted many attentions of the business and academic circles [3] [4]. However, the existing research focuses on tangible resources, but ignores the core 
knowledge that can build enterprise ability in the era of knowledge economy [5] [6]. Taking the development of the newly listed iPhone $\mathrm{X}$ as an example, Apple has concentrated on the design and research of its core technology, camera module researched by Sony Corporation, and assembled by Foxconn and other companies. Cooperation through various channels and parties (such as seminars, database and document sharing, team brainstorming, etc.) has finally completed the development of high-quality handsets. In the product development such as iPhone $\mathrm{X}$, which has complex knowledge base, high knowledge density and significant value of knowledge, cooperative enterprises mostly devote knowledge resources. Since the competitiveness of new products is more and more derived from the knowledge level, it is particularly important to focus on the investment of knowledge resources in the process of cooperated development.

New product development can be seen as a series of behavioral processes [7], Urban and Hauser [8] divided the new product development process into five phases: discovery of opportunities, product and process design, testing, commercialization, and late-stage feedback. New product development includes improvements to existing products and manufacturing of innovative products, and to improve existing products is the main $\mathrm{R} \& \mathrm{D}$ activities for most companies. In this process, the most challenging part is the product and process design, because this part not only need to integrate market demand into new products, but also consume a large amount costs, and even need to bear the huge risk of failure. Liu Hongwei [9] explored the composition of technology learning costs and its influencing factors, pointed out that the cost of technology learning mainly includes labor costs, technology prices and equipment investment and conversion costs. Through analyzing the literatures, Liu Hongwei points that the acquisition of knowledge resources among enterprises is a learning process, and the time and consumed resources in this process constitute the learning cost [10]. Therefore, this article will take the product and process design in cooperated development as an example to deeply explore the research questions.

For the supply chain, different company owns different knowledge resources; these heterogeneous and complementary knowledge resources formed a "supply chain knowledge base", which is obviously more abundant than any member in the supply chain [11]. If members in the supply chain participate in the new products development and fully share their knowledge base, the efficiency and competitiveness of the entire supply chain will be greatly enhanced, and each node enterprise can also benefit from knowledge sharing. However, because all the enterprises in the supply chain are different stakeholders and actors, and the sharing of knowledge resources requires huge costs and even faces the knowledge spillover which will weaken core competitiveness. Amaldoss and Rapopor [12] find that in the development alliance, potential learning has an impact on the investment behavior of cooperative enterprises. Ding and Huang [13] considered knowledge spillovers as one of the costs of collaborated knowledge creation in their research. Based on the existence of cost, Davenport and Prusak [14] 
put forward the concept of internal Knowledge market in "Working Knowledge" for the first time. He argues that there is a knowledge market within the enterprise which is similar to the commodity market, and the market mechanism can promote the flow and sharing of knowledge, as it does to physical goods. Therefore, this article will explore the research questions by using knowledge transaction as the cooperation mode between enterprises.

After Davenport and other scholars put forward the view of knowledge market, many scholars used this as a basis to study the knowledge transaction mechanism of knowledge exchange market in enterprises from different perspectives, such as quantitative and qualitative, which fully affirmed the promoting function of market trading mechanism to the knowledge transaction [15]. Some scholars think that the relationship between supply chain members is closer than other types of organizational alliances, such as strategic alliances, virtual enterprises, industry university research cooperation, etc., and think that it is more feasible to build a knowledge market in the supply chain [16]. Therefore, more scholars take the supply chain as the research background and explore by establishing Stackelberg model. For example, the domestic scholar Zhang Xumei [11] took the lead-agency theory as the quantitative analysis theory, and built the knowledge exchange market model under various factors, such as knowledge transaction enthusiasm, risk preference, transaction cost and external environment; Jiang Zhangsheng [17] used Stackelberg game model to explore the optimal knowledge transfer decision between alliance leader and follower in the innovation alliance; Wang Zhisheng [18] explored the knowledge transfer decision in R \& D Alliance on the basis of Stackelberg game model; Wu Jie [19] also used the Stackelberg game model to study the knowledge transfer strategy of all parties in the activities of enterprise-schoolinstitute. Therefore, the mature research method of the scholars should be used for reference. However, because tacit knowledge is difficult to code, manage and transfer, the existence of knowledge market manager will inevitably reduce the efficiency and effectiveness of knowledge transaction. Therefore, we think that direct knowledge transaction among enterprises in supply chain is the most effective way.

In view of the limitations of the existing research, this paper will take a manufacturer and its supplier in the supply chain as the research subject, and take the product and process design process in cooperated new product development as the research object, to explore the conditions for cooperation and the optimal resources investment decisions of both parties in the case of knowledge resources as the main investment resources. In particular, the flow of knowledge resources between the two parties takes place in knowledge transaction manner. This paper builds Stackelberg game model on the basis of in-depth analysis of knowledge transaction, and explores the resource investment decisions in this process. 


\section{Model Description and Hypotheses}

\subsection{Model Description}

The object of our research is to explore the optimal knowledge resources investment decisions and cooperative conditions for cooperated development of enterprises. In order to facilitate the modeling and analysis, we consider a two-echelon supply chain with a single manufacturer $(\mathrm{M})$ and a single supplier (S). Among them, the manufacturer $(\mathrm{M})$ is mainly responsible for product design, and supplier $(\mathrm{S})$ is mainly responsible for process design. Both companies in the cooperation process will selectively invest in knowledge resources, and the amount of investment resources will determine cooperation income. After the success of the new product development, the manufacturer will divide the cooperative income to the supplier according in a certain proportion. However, knowledge resources investment will bring certain cost, and the existence of knowledge spillover will weaken the core competitiveness of enterprises. Therefore, cooperative enterprises will choose the best resource investment decisions under the condition of maximizing their own interests.

The basic symbol definitions as follows:

$T$ : cooperative development cycle,

$A$ : ratio coefficient of cooperation income, constant every period,

$Q$ : value of new product,

$E:$ expected output,

$\mu$ : uncertainty parameters caused by the external environment,

$K_{M}$ : knowledge resources investment of manufacturer, $K_{M} \in[0,1]$,

$K_{S}$ : knowledge resources investment of supplier, $K_{S} \in[0,1]$,

$e_{M}$ : efforts degree of manufacturer, $e_{M} \in[0,1]$,

$e_{S}$ : efforts degree of supplier, $e_{S} \in[0,1]$,

$\lambda_{M}$ : influence coefficient of manufacturer's knowledge spillover,

$\lambda_{S}$ : influence coefficient of supplier's knowledge spillover,

$\theta_{M}$ : additional knowledge absorption rate of manufacturer, $\theta_{M} \in[0,1]$,

$\theta_{S}$ : additional knowledge absorption rate of supplier, $\theta_{S} \in[0,1]$,

$\pi_{M}$ : manufacturers' profits,

$\pi_{S}:$ suppliers' profits,

$\alpha:$ knowledge input-output elasticity of manufacturer, $\alpha \in(0,1)$,

$\beta$ : knowledge input-output elasticity of supplier, $\beta \in(0,1)$,

$\gamma:$ efforts degree input-output elasticity of manufacturer, $\gamma \in(0,1)$,

$\rho:$ efforts degree input-output elasticity of supplier, $\rho \in(0,1)$,

$\eta$ : the distribution proportion of the cooperative income, $\eta \in(0,1)$,

$c$ : the marginal cost of efforts degree,

$C:$ the total cost of efforts degree.

\subsection{Hypothesis of the Model}

Based on the above description of the problem, we have the following assumptions: 
Hypothesis 1: During the new products development process, the knowledge resources investment level of manufacturer and supplier respectively are $K_{M}, K_{S}$ and the efforts degree respectively are $e_{M}, e_{S}$. Where $e_{M}$ is constant, $K_{M}, K_{S}$ and $e_{S}$ are all personal information, $K \in[0,1], e \in[0,1]$

Hypothesis 2: With the development of knowledge transaction, the knowledge level of manufacturer and supplier has increased. As knowledge is embedded in new product, the value of new product is enhanced. We refer to the literature [13] to build knowledge production function $Q\left(K_{M}, K_{S}, e_{M}, e_{S}\right)=A K_{M}^{\alpha} K_{S}^{\beta} e_{M}^{\gamma} e_{S}^{\rho}+\mu$, and use it to represent the value of new products. Among them, $\mathrm{A}$ is a normal number, indicating the impact of factors such as the quality of the scientific and technical personnel and the system of the team on the output.

Hypothesis 3: $\mu$ indicates the uncertainty caused by the external environment, which is a normal distribution random variable with a mean value of 0 and a variance of $\sigma^{2}$.

Hypothesis 4: Referring to the model of literature [20], we set the total cost of resource investment as $C(e)=c T e$.

Hypothesis 5: Knowledge spillover is affected by knowledge investment, the absorptive capacity of receiver's knowledge and so on. We set up the cost of knowledge spillover as $\lambda_{M} K_{M}, \lambda_{S} K_{S}$. At the same time, enterprises can also gain additional benefits from the knowledge spillover from partners. Therefore, we respectively use $\theta_{M}, \theta_{S}$ to represent the knowledge absorbability of the manufacturer and the supplier for the extra income.

According to the model description and hypotheses, we can further obtain the profit of the manufacturer and the supplier:

The manufacturer's profit function is:

$$
\begin{aligned}
& \pi_{M}\left[\eta\left(K_{S}, e_{S}\right), K_{M}\left(K_{S}, e_{S}\right)\right] \\
& =\left(1-\eta+\theta_{M}\right) A K_{M}^{\alpha} K_{S}^{\beta} e_{M}^{\gamma} e_{S}^{\rho}-c_{M} T e_{M}-\lambda_{M} K_{M}
\end{aligned}
$$

The supplier's profit function is:

$$
\pi_{S}\left[K_{S}, e_{S} \mid \eta, K_{M}\right]=\left(\eta+\theta_{S}\right) A K_{M}^{\alpha} K_{S}^{\beta} e_{M}^{\gamma} e_{S}^{\rho}-c_{S} T e_{S}-\lambda_{S} K_{S}
$$

According to the above hypothesis, we can obtain the expected output:

$$
E(Q)=A K_{M}^{\alpha} K_{S}^{\beta} e_{M}^{\gamma} e_{S}^{\rho}
$$

\section{For manufacturer:}

The first partial derivatives of expected output to the knowledge resources investment level is:

$$
\frac{\partial E(Q)}{\partial K_{M}}=\alpha K_{M}^{\alpha-1} A K_{S}^{\beta} e_{M}^{\gamma} e_{S}^{\rho}>0
$$

The two partial derivatives of expected output to the knowledge resources investment level is:

$$
\frac{\partial^{2} E(Q)}{\partial K_{M}^{2}}=\alpha(\alpha-1) K_{M}^{\alpha-2} A K_{S}^{\beta} e_{M}^{\gamma} e_{S}^{\rho}<0
$$




\section{For supplier:}

The first partial derivatives of expected output to the knowledge resources investment level is:

$$
\frac{\partial E(Q)}{\partial K_{S}}=\beta K_{M}^{\alpha} A K_{S}^{\beta-1} e_{M}^{\gamma} e_{S}^{\rho}>0
$$

The two partial derivatives of expected output to the knowledge resources investment level is:

$$
\frac{\partial^{2} E(Q)}{\partial K_{S}^{2}}=\beta(\beta-1) K_{M}^{\alpha} A K_{S}^{\beta-2} e_{M}^{\gamma} e_{S}^{\rho}<0
$$

According to the Equation (4), (5), (6), (7), we can conclude that expected output is a monotonous increasing function of knowledge investment level and efforts degree, and satisfies the law of diminishing marginal revenue.

\section{Cooperated Development Model}

Based on the above hypothesis, the knowledge transaction model of manufacturer and supplier is in accordance with the Stackelberg master-slave game model, and their decisions will be divided into two stages. In the first stage, the manufacturer decides his knowledge investment level and the proportion of profits allocated to the supplier. In the second stage, the supplier decides his knowledge investment level and efforts degree according to the manufacturer's decision.

\subsection{The Analyses of the Model}

We use reverse induction to solve the model. First of all, the manufacturer needs to know the optimal decision of the supplier, and then make its own profit maximization decision under the optimal decision of the supplier. Therefore, Suppliers make their own optimal decisions

According to the reverse induction method and the optimality condition, the supplier's knowledge investment level $K_{S}$ and the supplier's efforts degree $e_{S}$ must satisfy the following conditions:

$$
\frac{\partial \pi_{S}}{\partial K_{S}}=\beta K_{S}^{\beta-1}\left(\eta+\theta_{S}\right) A K_{M}^{\alpha} e_{M}^{\gamma} e_{S}^{\rho}-\lambda_{S}=0
$$

and

$$
\frac{\partial \pi_{S}}{\partial e_{S}}=\rho e_{S}^{\rho-1}\left(\eta+\theta_{S}\right) A K_{M}^{\alpha} K_{S}^{\beta} e_{M}^{\gamma}-\left(c_{S} T\right)=0
$$

when the simultaneous Equations (8) and (9) are solved, the optimal value of $K_{S}$ and $e_{S}$ can be satisfied:

$$
K_{S}^{*}=\left[\frac{\left(c_{S} T\right)^{\rho} \cdot \beta^{\rho-1}}{\rho^{\rho} \lambda_{S}^{\rho-1}\left(\eta+\theta_{S}\right) A e_{M}^{\gamma} K_{M}^{\alpha}}\right]^{\frac{1}{\rho+\beta-1}}
$$

and 


$$
e_{s}^{*}=\left[\frac{\lambda_{S}^{\beta} \rho^{\beta-1}}{\beta^{\beta}\left(c_{S} T\right)^{\beta-1}\left(\eta+\theta_{S}\right) A e_{M}^{\gamma} K_{M}^{\alpha}}\right]^{\frac{1}{\rho+\beta-1}}
$$

Therefore, we obtain the optimal knowledge resources investment decision $K_{S}^{*}$ and optimal efforts degree $e_{s}^{*}$ of supplier.

For the manufacturer, he needs to optimize own interests according to the supplier's decisions. Therefore, we need to get profit function of the manufacturer according to $K_{S}^{*}$ and $e_{s}^{*}$, and the obtain the decision conditions of the manufacturer when he has the maximum value.

To simplify the expression, we do the following Settings:

$$
E=\left[\frac{\left(c_{S} T\right)^{\rho} \beta^{\rho-1}}{\rho^{\rho} \lambda_{S}^{\rho-1}\left(\eta+\theta_{S}\right) A e_{M}^{\gamma} K_{M}^{\alpha}}\right]^{\frac{1}{\rho+\beta-1}}
$$

and

$$
D=\left[\frac{\lambda_{S}^{\beta} \rho^{\beta-1}}{\beta^{\beta}\left(c_{S} T\right)^{\beta-1}\left(\eta+\theta_{S}\right) A e_{M}^{\gamma} K_{M}^{\alpha}}\right]^{\frac{1}{\rho+\beta-1}}
$$

Then, take the Equation (12), (13) into the Equation (1), we can get the simplified expression of $\pi_{M}$ :

$$
\pi_{M}\left(\eta, K_{M}\right)=\left(1-\eta+\theta_{M}\right) A K_{M}^{\alpha} E^{\beta} e_{M}^{\gamma} D^{\rho}-c_{M} T e_{M}-\lambda_{M} K_{M}
$$

when $t=\frac{-1}{\beta+\rho-1}, \pi_{M}$ can be expressed as:

$$
\pi_{M}\left(\eta, K_{M}\right)=\left(1+\theta_{M}-\eta\right)\left(\eta+\theta_{S}\right)^{-1+t} A^{t} K_{M}^{\alpha t} e_{M}^{\gamma t}\left(\frac{c_{S} T \lambda_{S}}{\beta \rho}\right)^{-t}
$$

For the manufacturer, If we want to find the maximum value of $\pi_{M}$, we need to find the optimal value of $K_{M}$ and $\eta$ firstly.

Therefore, we respectively get the first-order partial derivatives of $K_{M}$ and $\eta$ to $\pi_{M}$ as following:

$$
\begin{aligned}
& \frac{\partial \pi_{M}}{\partial K_{M}}=(\alpha t) K_{M}^{\alpha t-1} A^{t} e_{M}^{\gamma t}\left(\frac{c_{S} T \lambda_{s}}{\beta \rho}\right)^{-t}\left(1-\eta+\theta_{M}\right)\left(\eta+\theta_{S}\right)^{-1+t}-\lambda_{M} \\
& \frac{\partial \pi_{M}}{\partial \eta}=K_{M}^{\alpha t} A^{t} e_{M}^{\gamma t}\left[-\left(\eta+\theta_{S}\right)^{-1+t}+(-1+t)\left(\eta+\theta_{S}\right)^{-2+t}\left(1-\eta+\theta_{M}\right)\right]
\end{aligned}
$$

By taking $\frac{\partial \pi_{M}}{\partial K_{M}}=0$ and $\frac{\partial \pi_{M}}{\partial \eta}=0$, we have:

$$
K_{M}^{*}=\left\{\begin{array}{l}
{\left[\frac{\lambda_{M}}{\alpha\left(1+\theta_{M}+\theta_{S}\right) A^{t} e_{M}^{\gamma t}}\right]^{\frac{1}{\alpha t-1}}\left(\frac{\beta \rho}{c_{S} T \lambda_{s}}\right)^{\frac{-t}{\alpha t-1}}} \\
\cdot\left[\frac{(1-t)\left(1+\theta_{M}-\theta_{S}\right)}{t}\right]^{\frac{1-t}{\alpha t-1}}, \frac{1+\theta_{M}+\theta_{S}}{2+2 \theta_{M}+\theta_{S}}<\beta+\rho<1 \\
0, \quad \text { others }
\end{array}\right.
$$


and

$$
\eta^{*}=\left\{\begin{array}{lc}
\frac{\theta_{S}+(1-t)\left(1+\theta_{M}\right)}{-t}, & \frac{1+\theta_{M}+\theta_{S}}{2+2 \theta_{M}+\theta_{S}}<\beta+\rho<1 \\
0, & \text { others }
\end{array}\right.
$$

Under the condition of the manufacturer's maximization, we can reverse the supplier's maximization condition.

Similarly, in order to simplify the expression, we have:

$$
\begin{gathered}
G=\frac{\left(c_{S} T\right)^{\rho} \beta^{\rho-1}}{\rho^{\rho} \lambda_{S}^{\rho-1} A e_{M}^{\gamma}} \\
H=\frac{\lambda_{S}^{\beta} \rho^{\beta-1}}{\beta^{\beta}\left(c_{S} T\right)^{\beta-1} A e_{M}^{\gamma}} \\
J=\frac{\theta_{S}+(1-t)\left(1+\theta_{M}\right)}{-t}
\end{gathered}
$$

Substituting Equations (20), (21) and (22) into the Equation of (10) and Equation of (11), we have:

$$
\begin{gathered}
K_{S}^{*}=G^{-t}\left(J+\theta_{S}\right)^{t} M^{\alpha t} \\
e_{S}^{*}=H^{-t}\left(J+\theta_{S}\right)^{t} M^{\alpha t}
\end{gathered}
$$

Hence, we obtain the optimal knowledge investment decision and efforts degree of the manufacturer and the supplier.

\subsection{Model Propositions}

Based on the above analysis, we have the following proposition:

\section{Proposition 1}

The cooperation relationship can be established only when the knowledge investment level and the elasticity of the efforts degree of the supplier are greater than $\frac{1+\theta_{M}+\theta_{S}}{2+2 \theta_{M}+\theta_{S}}$.

Proof of proposition 1

When analyze the optimal proportion of income distribution value, we can find that if $\eta^{*}>0, \beta+\rho$ must meet the conditions that $\beta+\rho \in\left(\frac{1+\theta_{M}+\theta_{S}}{2+2 \theta_{M}+\theta_{S}}, 1\right)$.

The supplier's knowledge investment level and the elasticity coefficient of the effort degree reflect the ability of the supplier to develop new products. Lower elasticity coefficient will get less output. This kind of situation indicates the weak development capability of the supplier, and represents the high risk of failure or lower profit when manufacturer cooperates with it. As a result, the manufacturer should choose to cooperate with the supplier who has certain development capabilities.

Proposition 2 
When $\frac{1+\theta_{M}+\theta_{S}}{2+2 \theta_{M}+\theta_{S}}<\beta+\rho<1$ and $\frac{1}{\alpha t-1}<0$, the knowledge investment level by a manufacturer will increase as its extra rate of return yield increases.

Proof of proposition 2

When $\frac{1+\theta_{M}+\theta_{S}}{2+2 \theta_{M}+\theta_{S}}<\beta+\rho<1$ and $\frac{1}{\alpha t-1}<0$, we have $\frac{\partial K_{M}^{*}}{\partial \theta_{M}}>0$, Which show that the knowledge investment level by a manufacturer will increase as its extra rate of return yield increases.

Through the analysis of $K_{M}^{*}$, we can see that the knowledge investment level of the manufacturer is an increasing function of the extra rate of return. Due to the existence of knowledge spillover phenomenon, knowledge acceptor can absorb knowledge of partners through learning behavior, thus increasing knowledge stock and improving their competitiveness to a certain extent. Therefore, if the extra profit of the manufacturer is higher, he will be more willing to maintain cooperation, so he will invest more knowledge resources to express the willingness to cooperate.

\section{Proposition 3}

When $\frac{1+\theta_{M}+\theta_{S}}{2+2 \theta_{M}+\theta_{S}}<\beta+\rho<1$ and $\frac{1}{\alpha t-1}<0$, the supplier's effort degree will decrease with the increase of the extra rate of return.

Proof of proposition 2

When $\frac{1+\theta_{M}+\theta_{S}}{2+2 \theta_{M}+\theta_{S}}<\beta+\rho<1$ and $\frac{1}{\alpha t-1}<0$, we have $\frac{\partial e_{S}^{*}}{\partial \theta_{S}}>0$, Which show that the supplier's effort degree will decrease with the increase of the extra rate of return.

Through the analysis of $e_{S}^{*}$, we can see that the efforts degree of the supplier is a decreasing function of the extra rate of return. This conclusion may be due to that efforts cost a certain cost, and suppliers will get more extra benefits with the increase of extra absorption rate in the cooperation process. At the same time, as the degree of effort is an unobservable variable, the supplier may have the phenomenon of hitchhiking in order to save cost. As a result, the supplier's effort degree will decrease with the increase of the extra rate of return.

\section{Numerical Example Analysis}

Finally, a numerical example is presented to analyze this knowledge transaction.

\subsection{The Impact of Knowledge Spillover Level to the Knowledge Investment Level}

In order to explore the impact of manufacturer's level of knowledge spillover on the knowledge investment level, we assignments for the relevant parameters as $\theta_{M}=0.2, \quad \theta_{S}=0.3, \alpha=0.1, \quad \beta=0.4, \rho=0.4, \gamma=0.4, A=1, e_{M}=0.2$, $c_{s}=0.1, T=1, \lambda_{s}=0.2$. Meanwhile we have $\lambda_{M} \in[0,1]$. The result is shown in Figure 1. 


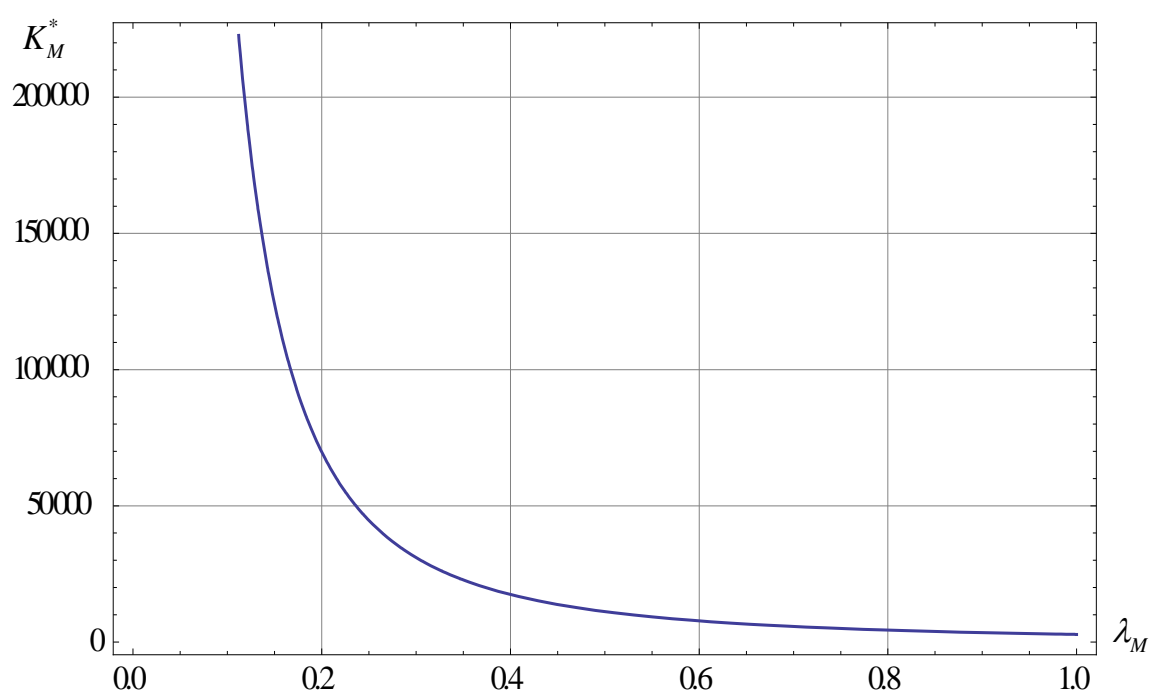

Figure 1. The impact of knowledge spillover level $\lambda_{M}$ to the knowledge investment level $K_{M}^{*}$.

According to Figure 1, we can see that when $\lambda_{M}<0.3$, the change rate of $K_{M}^{*}$ is relatively obvious. This situation shows that in the case of low level of knowledge spillover, with the decline of knowledge spillover, manufacturers are more willing to invest in knowledge resources for new product development. when $\lambda_{M}>0.4$, the change rate of $K_{M}^{*}$ is not obvious. Because the existence of knowledge spillover, the manufacturer is reluctant to invest too much knowledge.

As can be seen from Figure 1 and the above analysis, the knowledge spillover level has a great impact on knowledge resources investment decision. The knowledge spillover level represents the risk that the core competitiveness will be weakened caused by the investment of knowledge resources. However, the core competition of enterprise is the foundation of its development and the source of its development. Therefore, in order for cooperation and development to be carried out smoothly, cooperated enterprises should establish knowledge protection mechanisms, such as signing contracts and applying for property rights protection, so as to avoid risks and promote transactions.

\subsection{The Impact of Extra Rate of Return to the Knowledge Investment Level}

To explore the impact of manufacturer's extra rate of return on the knowledge investment level, we assignments for the relevant parameters as $\theta_{S}=0.3$, $\alpha=0.1, \quad \beta=0.4, \quad \rho=0.4, \quad \gamma=0.4, \quad A=1, \quad e_{M}=0.2, \quad c_{s}=0.1, \quad T=1$, $\lambda_{s}=0.2, \lambda_{M}=0.3$. Meanwhile we have $\theta_{M} \in[0,1]$. The result is shown in Figure 2 .

According to Figure 2, we can see that as the manufacturer's extra rate of return increases, its knowledge investment level also gradually increases. when $\theta_{M}>0.8, K_{M}^{*}$ changes significantly. Because in the case of higher extra rate of 


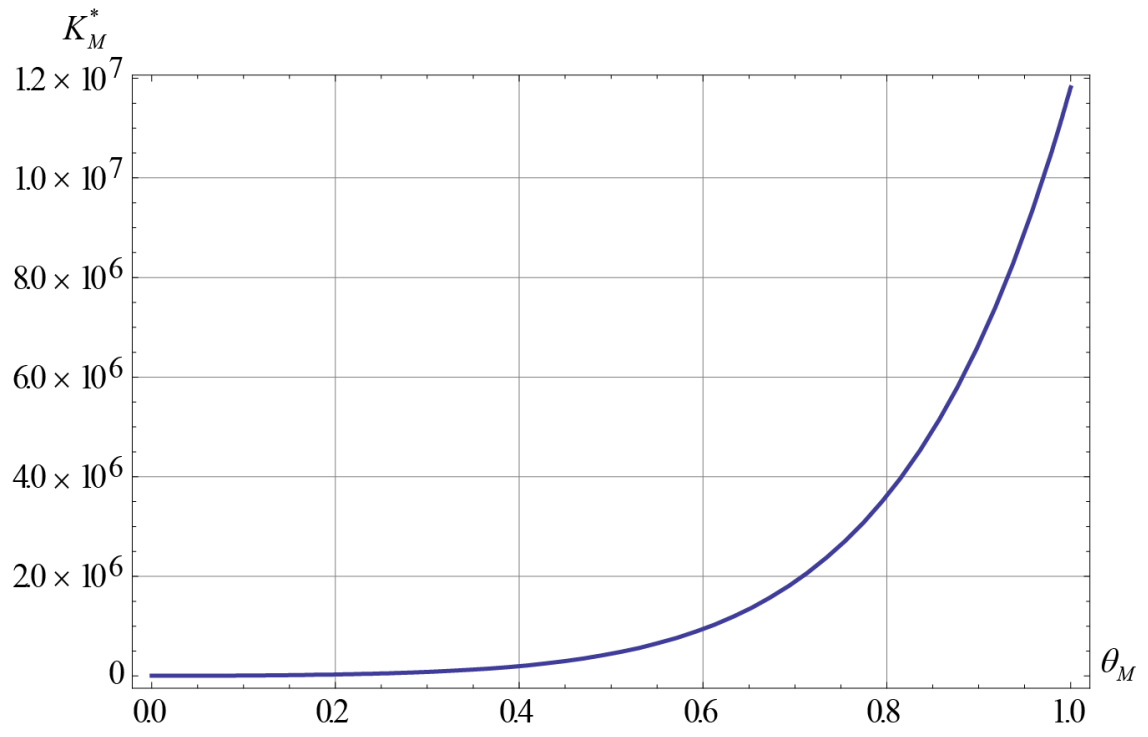

Figure 2. The impact of extra rate of return $\theta_{M}$ to the knowledge investment level $K_{M}^{*}$.

return, the manufacturer wants to gain other benefits in addition to new product benefits, such as reputation.

From the Figure 2 and above analysis, it can be shown that the extra rate of return has a great influence on the decision of the enterprise's knowledge resource investment. The extra rate of return represents the possibility that the enterprise obtains the extra income from the partner in the cooperation. When the extra rate of return is higher, the enterprise is more likely to gain more benefits from the cooperation, such as additional knowledge resources. Therefore, for manufacturers, when faced with a higher rate of extra return, they should actively invest resources and provide a substantial proportion of product returns to partners, so as to attract suppliers to participate in cooperation. And when the extra rate of return is low, manufacturers tend to be reluctant to invest in resources, which will make cooperation unsustainable. At this point, manufacturers should enhance their own extra rate of return by introducing professional talents, holding training meetings and strengthening internal communication learning.

\subsection{The Impact of Extra Rate of Return to the Distribution Proportion of Income}

To explore the impact of supplier's extra rate of return on the distribution proportion of income, we assignments for the relevant parameters as $\theta_{M}=0.2$, $\alpha=0.1, \quad \beta=0.4, \quad \rho=0.4, \quad \gamma=0.4, \quad A=1, \quad e_{M}=0.2, \quad c_{s}=0.1, \quad T=1$, $\lambda_{s}=0.2, \lambda_{M}=0.3$. Meanwhile, we have $\theta_{S} \in[0,1]$. The result is shown in Figure 3.

According to Figure 3, we can see that with the increase of the supplier's extra rate of return, the distribution proportion of income gradually decreases. This is because, given the common knowledge, the extra rate of return of the supplier 


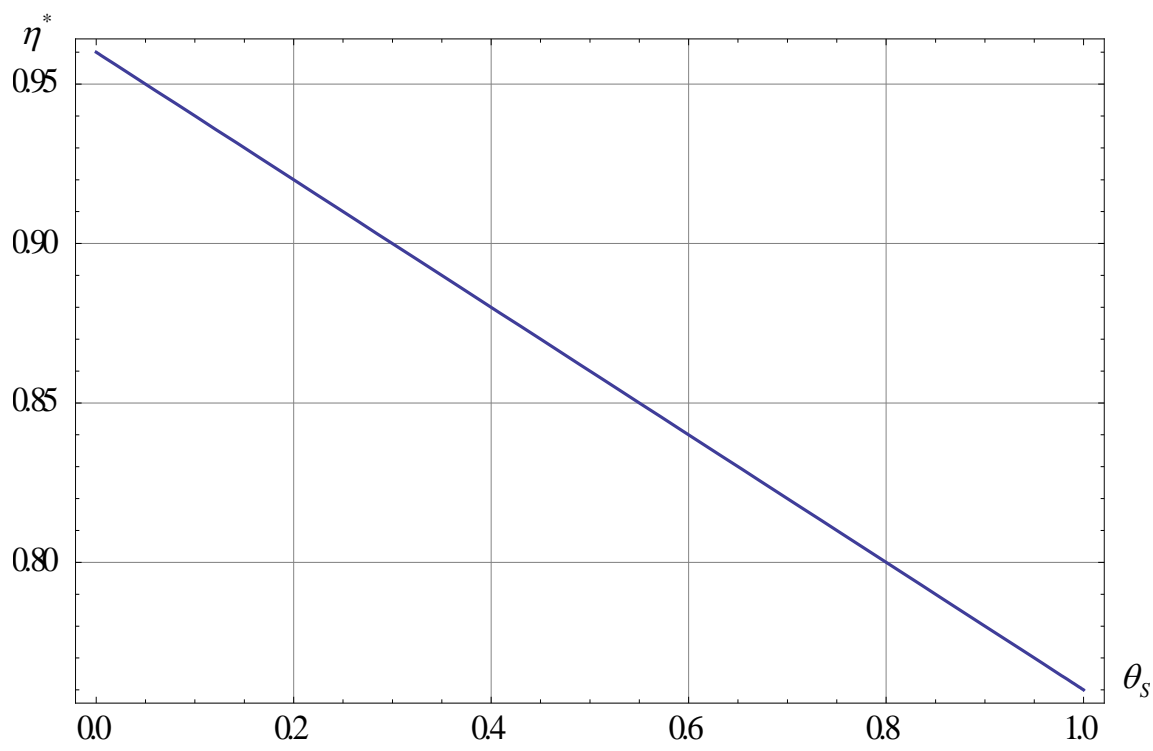

Figure 3. The impact of extra rate of return $\theta_{S}$ to the distribution proportion of income $\eta^{*}$.

will make the manufacturer think that his participation in the cooperative development is not for the purpose of obtaining the product benefit, but for obtaining other additional benefits. Therefore, the manufacturer will not allocate more revenue to the supplier.

From Figure 3 and above analysis, it can be seen that the extra rate of return of suppliers has a great impact on the decision of the manufacturer's income distribution. The extra rate of return of suppliers represents the possibility that the supplier can obtain additional benefits from the manufacturer in the cooperation, especially the acquisition of core knowledge resources. Therefore, the decision of the manufacturer's income distribution decreases with the increase of the supplier's extra return rate. Although the distribution proportion effectively restricts the profit grabbing of the supplier, it can't effectively protect the knowledge resources of the manufacturer. Therefore, when the supplier has a high extra rate of return, the manufacturer should actively sign the knowledge protection agreement with the supplier and make the corresponding knowledge protection policy.

The above three parts use numerical analysis method, and graphically shows the impact of manufacturer's knowledge spillover level $\lambda_{M}$ on knowledge investment level $K_{M}$, manufacturer's extra profit rate impact $\theta_{M}$ on knowledge input level $K_{M}$, and the effect of the extra rate of return $\theta_{S}$ on the manufacturer's profit distribution decision $\eta^{*}$. At the same time, we also come to the conclusion through analysis, and put forward the practical guidance of the enterprise.

\section{Discuss and Conclusions}

In the existing research on the cooperated new product development, knowledge 
has been considered as a general element. However, in the era of knowledge economy, the value of knowledge resources to the product has far exceeded the entity resources. Based on this situation, we explore the partners' decisions about knowledge resources investment, efforts degree and the distribution proportion of income among partners.

Based on the analysis of Stackelberg's Master-Slave game model, we obtain the optimal decision of different enterprise. At the same time, we discuss the influence of different factors on the management decisions of both sides, and obtain the following conclusions:

First, the knowledge spillover level influences knowledge resources investment decision. The higher the knowledge spillover level, the lower the willingness of manufacturers to invest their knowledge resources. Therefore, in order for cooperation and development to be carried out smoothly, cooperated enterprises should establish knowledge protection mechanisms, such as signing contracts and applying for property rights protection, so as to avoid risks and promote transactions.

Second, the extra rate of return has a great influence on the decision of the enterprise's knowledge resource investment. The extra rate of return represents the possibility that the enterprise obtains the extra income from the partner in the cooperation. Therefore, for manufacturers, in order to attract suppliers to participate in the cooperation when they have higher extra rate of return, they should take the initiative to invest resources and provide considerable proportion of product revenue distribution to the partners. When the extra rate of return is low, manufacturers tend to be reluctant to invest in resources, which will make cooperation unsustainable. At this point, manufacturers should enhance their own extra rate of return by introducing professional talents, holding training meetings and strengthening internal communication learning.

Third, the extra rate of return of suppliers has a great impact on the decision of the manufacturer's income distribution. The extra rate of return of suppliers represents the possibility that the supplier can obtain additional benefits from the manufacturer in the cooperation, especially the acquisition of core knowledge resources. Although the distribution proportion effectively restricts the profit grabbing of the supplier, it can't effectively protect the knowledge resources of the manufacturer. Therefore, when the supplier has a high extra rate of return, the manufacturer should actively sign the knowledge protection agreement with the supplier and make the corresponding knowledge protection policy.

The decision model and relevant conclusions proposed in this paper can provide some guidance to the enterprise.

Finally, we need to point out that we assume that both parties involved in cooperated development are risk neutral and limited rational, which couldn't include all business practices. In the actual cooperated development, there may be a phenomenon that one party will terminate the cooperation or the new product 
development will fail. In addition, the new product development process is actually a dynamic process, and the knowledge investment of both sides may change over time. Based on this reality, follow-up research can be conducted from the following aspects: 1) consider the dynamic investment of knowledge resources; 2) consider the failure of cooperated new product development.

\section{References}

[1] Bhaskaran, S.R. and Krishnan, V. (2009) Effort, Revenue, and Cost Sharing Mechanisms for Collaborative New Product Development. Management Science, 55, 1152-1169. https://doi.org/10.1287/mnsc.1090.1010

[2] Grant, R.M. (1996) Toward a Knowledge-Base Theory of the Firm.

[3] Fu, J.H., Zhang, L. and Cheng, Z.M. (2016) Substitution Degree of the Product, Knowledge Sharing and Enterprise R\&D Cooperation. Journal of Industrial Engineering and Engineering Management, 1, 1-8.

[4] Li, X.Y. and Feng, J.W. (2011) Optimization of New Product Development Process Based DEA. Systems Engineering Theory Methodology Applications, 3, 356-362.

[5] Zhu, X.M., Jiang, Y., Du, Z.W., et al. (2011) A Study on the Impact of KM Processes on New Product Development Performance. Journal of Industrial Engineering and Engineering Management, 4, 113-122.

[6] Chen, Z.X., Chen, R.Q. and Ma, S.H. (2000) Theory of Knowledge Chain and Knowledge Management. Science Research Management, 1, 14-18.

[7] Yin, J. and Peng, C. (2008) The Knowledge Management of the New Product Development Process. Technoeconomics \& Management Research, 6, 34-36.

[8] Rban, G.H.J. (1993) Design and Marketing of New Products. Prentice Hall.

[9] Liu, H.W., Li, J.Z. and Wang, Y. (2007) An Analysis on the Cost of Technological Learning and Its Major Determinates. Science Research Management, 5, 1-8.

[10] Liu, H.W., Wu, G.S. and He, J.S. (2009) Knowledge and Information: Learning Cost and Transactional Cost. Technology Economics, 9, 5-11.

[11] Zhang, X.M., Zhang, Y.R., Zhu, Q., et al. (2008) Study on the Knowledge Market and the Model of Knowledge Trading among Enterprises in Supply Chain. Journal of Industrial Engineering and Engineering Management, 3, 79-83.

[12] Amaldoss, W. and Rapoport, A. (2005) Collaborative Product and Market Development: Theoretical Implications and Experimental Evidence. Marketing Science, 24, 396-414. https://doi.org/10.1287/mksc.1040.0105

[13] Ding, X.H. and Huang, R.H. (2010) Effects of Knowledge Spillover on Inter-Organizational Resource Sharing Decision in Collaborative Knowledge Creation. European Journal of Operational Research, 201, 949-959.

https://doi.org/10.1016/j.ejor.2009.04.008

[14] Davenport, T.H. and Prusak, L. (1998) Working Knowledge. Harvard Business School Press, Boston, MA.

[15] Ba, S., Stallaert, J. and Whinston, A.B. (2001) Optimal Investment in Knowledge within a Firm Using a Market Mechanism. Management Science, 9, 1203-1219. https://doi.org/10.1287/mnsc.47.9.1203.9781

[16] Chen, W. and Song, H. (2014) Optimal Contract Configuration of Knowledge Trading among Enterprises in Supply Chain Considering Influence of Learning Capability. Technology Economics, 1, 125-133.

[17] Jiang, Z.S. and Hu, L.Y. (2012) The Knowledge Transfer Decisions in a Technology 
Innovation Alliance Based on Leader-Followers Game. Science Research Management, 4, 41-47.

[18] Wang, Z.S. and Li, H.Y. (2016) A Knowledge Transfer Decision Model of R\&D Alliance Based on the Stackelberg Game. Science Research Management, 6, 74-83.

[19] Wu, J., Peng, X.X., Sheng, Y.X., et al. (2017) Research on Cooperative Game of Knowledge Transfer in Industry, University and Research Based on Dynamic Control Model. Chinese Journal of Management Science, 3, 190-196.

[20] Xiong, Y., Zhang, X.B. and Xiong, Z.K. (2013) Capital and Knowledge Input Decision on Collaborative New Product Development. Journal of Management Sciences in China, 9, 53-63. 